FERMILAB-Conf-97/165-E

CDF and DØ

\title{
B Physics at the Tevatron Collider
}

\author{
Jorge F. De Troconiz \\ For the CDF and D $\varnothing$ Collaborations \\ Harvard University \\ Cambridge, Massachusetts 02138
}

Fermi National Accelerator Laboratory

P.O. Box 500, Batavia, Illinois 60510

May 1997

To be Published in the Proceedings of the XXXIInd Rencontres de Moriond, Electroweak Interactions and Unified Theories, Les Arcs, France, March 22-29, 1997 


\section{Disclaimer}

This report was prepared as an account of work sponsored by an agency of the United States Government. Neither the United States Government nor any agency thereof, nor any of their employees, makes any warranty, expressed or implied, or assumes any legal liability or responsibility for the accuracy, completeness, or usefulness of any information, apparatus, product, or process disclosed, or represents that its use would not infringe privately owned rights. Reference herein to any specific commercial product, process, or service by trade name, trademark, manufacturer, or otherwise, does not necessarily constitute or imply its endorsement, recommendation, or favoring by the United States Government or any agency thereof. The views and opinions of authors expressed herein do not necessarily state or reflect those of the United States Government or any agency thereof.

\section{Distribution}

Approved for public release; further dissemination unlimited. 


\title{
B PHYSICS AT THE TEVATRON COLLIDER *
}

JORGE F. DE TROCONIZ ${ }^{\dagger}$

Harvard University, 42 Oxford Street

Cambridge, Massachusetts 02138, USA

\begin{abstract}
Precision $B$-physics results from the CDF and D0 Collaborations based on data collected during the Tevatron 1992-96 run are presented. In particular we discuss the measurement of $B$ hadron lifetimes, and $B^{0}-\bar{B}^{0}$ mixing results obtained using time-evolution analyses. Perspectives for the next Tevatron run, starting in 1999 , are also reported.
\end{abstract}

\section{Introduction}

Precision $B$ physics has become possible at hadron colliders since the CDF Collaboration installed a silicon vertex detector (SVX) in 1992. The total $b$ production cross section at the Fermilab Tevatron is about $30 \mu \mathrm{b}^{1}$ in the rapidity region $|y|<1$. For a typical instantaneous luminosity of $10^{31} \mathrm{~cm}^{-2} \cdot \mathrm{sec}^{-1}$ the corresponding production rate is $300 \mathrm{~Hz}$. However the backgrounds are also large: the $b$ cross section is three orders of magnitude smaller than the total inelastic cross section. Clearly the trigger performance is of vital importance. All $B$ triggers at hadron colliders are based on leptons.

The CDF and D0 Collaborations have collected $100 \mathrm{pb}^{-1}$ of data during the 1992-96 run. The results discussed here are based on these data. In the following we shall report the latest CDF results on the measurement of $B$ hadron lifetimes, and time-dependent $B^{0}-\bar{B}^{0}$ mixing.

\section{B Hadron Lifetimes}

The $B$ hadron lifetimes are sensitive to the details of the decay mechanism beyond the spectator model. Unlike the $D^{+} / D^{0}$ case, $B$ decay models predict very small differences between the $B_{u}$ and the $B_{d}$ lifetimes $(5-10 \%)^{2,3}$. Currently there is some controversy among theorists about the precise size of the effect. Sensitivities of this order of magnitude or smaller are

*To be published in the Proceedings of the XXXIInd Rencontres de Moriond, Electroweak Interactions and Unified Theories, Les Arcs, France, March 1997

${ }^{\dagger}$ Representing the CDF and D0 Collaborations 
therefore necessary in order to constrain the theory.

CDF reports an update on the exclusive $B_{u}$ and $B_{d}$ lifetimes, which uses a total of $110 \mathrm{pb}^{-1}$ of data. There is a new measurement of these lifetimes using semi-exclusive channels. The $B_{s}$ lifetime has also been measured using the decay mode $B_{s} \rightarrow l \nu D_{s}$. Finally, the $\Lambda_{b}$ has been measured using the decay $\Lambda_{b} \rightarrow l \nu \Lambda_{c}$.

\subsection{Exclusive $B^{+}$and $B^{0}$ Lifetimes}

This measurement of the $B$ meson lifetimes uses their exclusive decays into $J / \psi^{4}$. The analysis selects decays $J / \psi \rightarrow \mu^{+} \mu^{-}$from a dimuon trigger data sample. The two opposite charge muons are constrained to originate from a common vertex and the invariant mass is calculated. Approximately 240,000 $\mathrm{J} / \psi$ 's with both legs reconstructed in the SVX are found with less than $10 \%$ background in the peak region.

$J / \psi$ candidates are then associated to additional tracks to select specific $B$ decay modes. The $B$ decay modes used for this analysis are: $B \rightarrow \Psi \mathbf{K}$, where $\Psi$ is either a $J / \psi$ or a $\psi(2 S)$ and $\mathbf{K}$ can be any of the following: $K^{ \pm}, K^{* 0} \rightarrow K \pi, K_{S}^{0} \rightarrow \pi^{+} \pi^{-}$or $K^{*+} \rightarrow K_{S}^{0} \pi^{+}$.

We define the transverse decay distance, $L_{x y}$, of the $B$ meson as the vector difference of the secondary and primary vertex positions in the transverse plane projected onto the $B$ transverse momentum vector, $\vec{p}_{t}^{B}$ :

$$
L_{x y}=\frac{\left(\vec{x}_{s e c}-\vec{x}_{p r i m}\right) \cdot \vec{p}_{t}^{B}}{p_{t}^{B}}
$$

From $L_{x y}$ we extract the proper decay length, $c \tau$, by correcting with the appropriate $\beta_{t} \gamma$ factor:

$$
c \tau=L_{x y} \frac{M_{B}}{p_{t}^{B}}
$$

where $M_{B}$ is the mass of the $B$ meson and $p_{t}^{B}$ its transverse momentum.

With these definitions, the proper decay time distributions for charged and neutral $B$ mesons are calculated. The signal region is defined within $\pm 30 \mathrm{MeV} / \mathrm{c}^{2}$ of the world average $B$ mass. Similarly, a sideband region is defined by the condition $60 \mathrm{MeV} / \mathrm{c}^{2}<|\Delta M|<120 \mathrm{MeV} / \mathrm{c}^{2}$. The $c \tau$ distributions for charged and neutral $B$ 's in the two mass regions are shown in Figure 1. The shape of the background is determined by fitting the sideband distribution to a gaussian with exponential tails. The shape of the signal region is assumed to be a sum of the sideband shape and an exponential convoluted with a gaussian resolution function. After performing an unbinned fit (to account for the event by event variation of the $L_{x y}$ resolution) the following results are obtained:

$$
\begin{gathered}
\tau^{+}=1.68 \pm 0.07 \text { (stat.) } \pm 0.02 \text { (syst.) ps } \\
\tau^{0}=1.58 \pm 0.09 \text { (stat.) } \pm 0.02(\text { syst. }) \mathrm{ps} \\
\tau^{+} / \tau^{0}=1.06 \pm 0.07 \text { (stat.) } \pm 0.01 \text { (syst.) }
\end{gathered}
$$

The largest systematic error is due to the shape of the background distribution. However, this measurement is still statistics dominated. One way to increase the number of candidates is to reconstruct partially the $B$ meson. This method is discussed in the next section.

\subsection{Semi-exclusive $B^{+}$and $B^{0}$ Lifetimes}

Another approach to measure $B_{u, d}$ lifetimes is to use $B$ semileptonic decays ${ }^{5}$. Starting with a large sample of inclusive single lepton triggers, $D$ mesons are reconstructed in a cone around the trigger lepton using the following channels: 

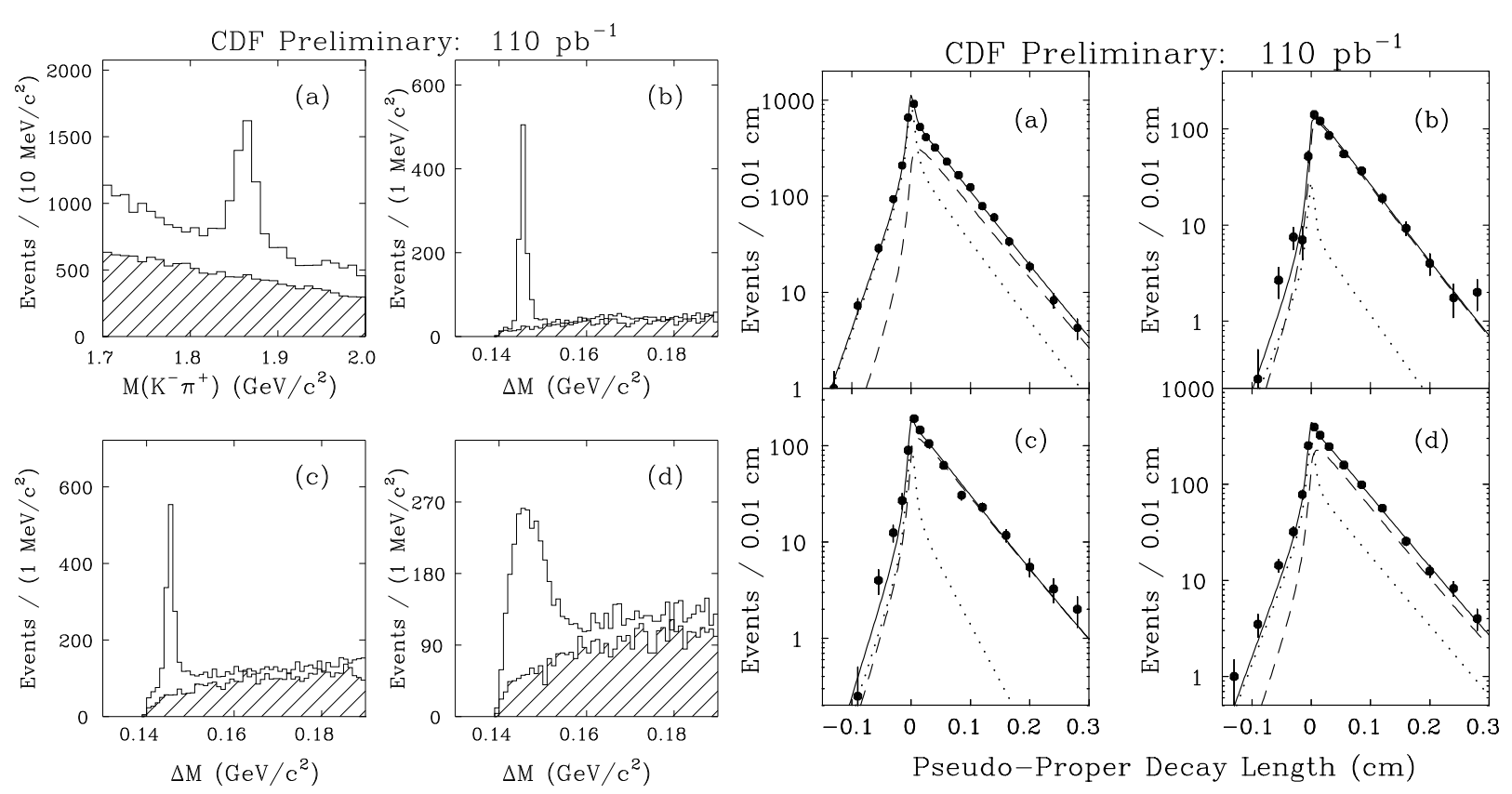

Fig. 2. Charm invariant mass peaks (left) and proper time distributions (right) used in the semiexclusive $B$ lifetime analysis: (a) $D^{0} \rightarrow K^{-} \pi^{+}$, (b) $D^{*+} \rightarrow D^{0} \pi^{+}, D^{0} \rightarrow K^{-} \pi^{+}$, (c) $D^{*+} \rightarrow$ $D^{0} \pi^{+}, D^{0} \rightarrow K^{-} \pi^{+} \pi^{+} \pi^{-}$, (d) $D^{*+} \rightarrow D^{0} \pi^{+}, D^{0} \rightarrow K^{-} \pi^{+} \pi^{0}$

These results are consistent with the ones obtained in the exclusive analysis. Combining them, the following lifetime averages are produced:

$$
\begin{gathered}
\tau^{+}=1.66 \pm 0.05 \mathrm{ps} \\
\tau^{0}=1.52 \pm 0.06 \mathrm{ps} \\
\tau^{+} / \boldsymbol{\tau}^{0}=1.09 \pm 0.05
\end{gathered}
$$

These numbers are competitive with the values obtained by the LEP and SLC experiments 6.

\section{3. $B_{s}$ Lifetime}

The lifetime of the $B_{s}$ meson has been measured ${ }^{7}$ using the semi-exclusive decay to $D_{s} l \nu$ with $D_{s} \rightarrow \phi \pi$ and $\phi \rightarrow K^{+} K^{-}$. The analysis techniques are similar to those described in the previous section. The left plot in Figure 3 shows a signal of $254 \pm 21$ "right sign" $D_{s}$ candidates above background. From them, a lifetime of

$$
\tau\left(B_{s}\right)=1.37_{-0.12}^{+0.14} \text { (stat.) } \pm 0.04 \text { (syst.) ps }
$$

is obtained. This measurement is still statistically limited.

\section{4. $\Lambda_{b}^{0}$ Lifetime}

The analysis to measure the $\Lambda_{b}$ lifetime is very similar to the previous ones. The $\Lambda_{b}$ baryon is reconstructed through the semileptonic decay $\Lambda_{b} \rightarrow \Lambda_{c} l \nu X$, where $\Lambda_{c} \rightarrow p K^{-} \pi^{+}{ }^{8}$. The analysis uses single lepton trigger data, looking for a $\Lambda_{c}$ candidate in a cone around the lepton. The $\Lambda_{b}$ decay vertex is found at the intersection of the $\Lambda_{c}$ and lepton decay lines. Using a signal of $197 \pm 25$ right sign candidates (Figure 3 (right)), a lifetime of

$$
\tau\left(\Lambda_{b}^{0}\right)=1.32 \pm 0.15 \text { (stat.) } \pm 0.07 \text { (syst.) ps }
$$



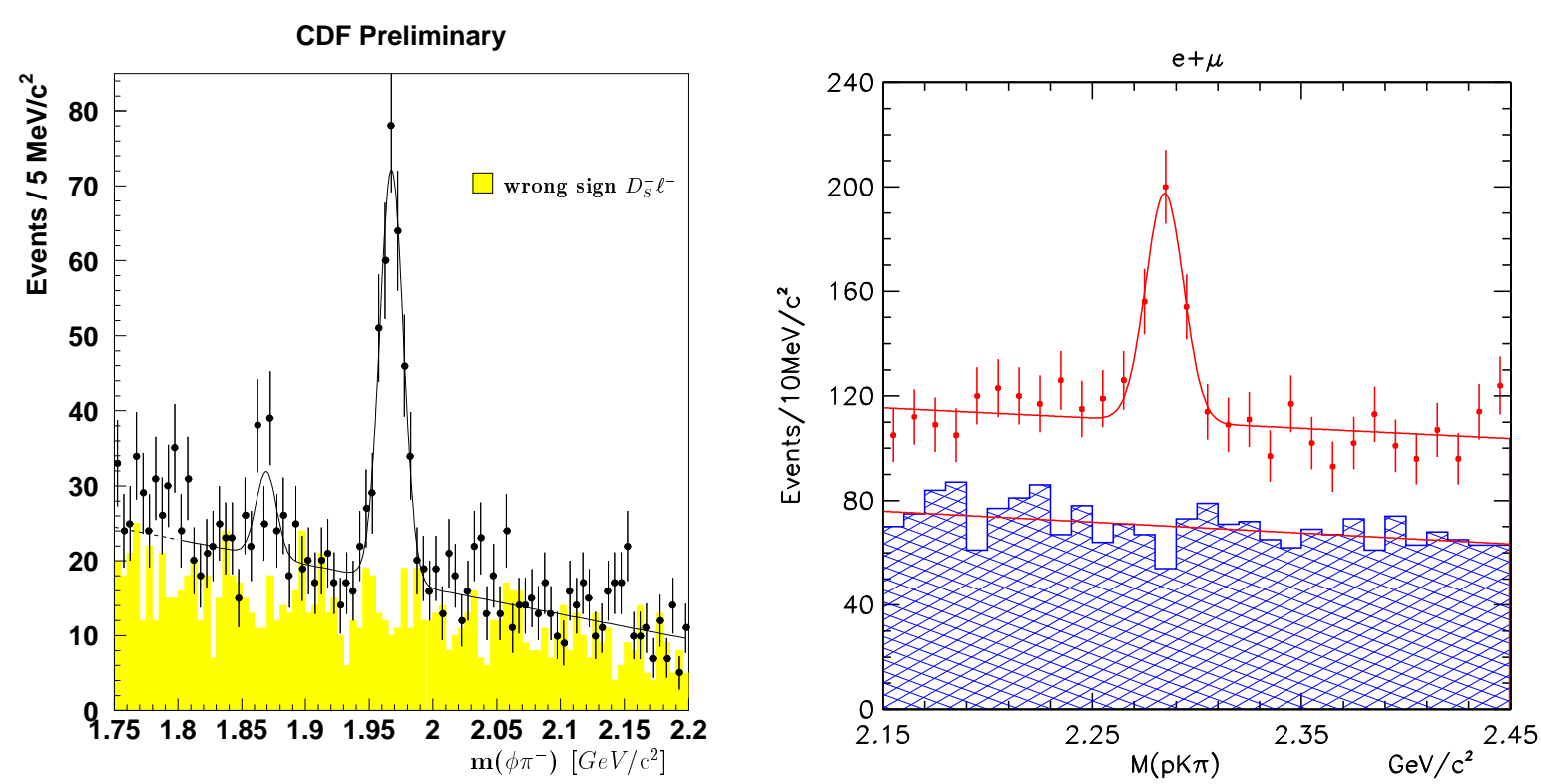

Fig. 3. (Left) Invariant mass of the $\phi \pi$ system. The points are for right sign $l D_{s}$ combinations. The shaded histogram shows the wrong sign combinations distribution. (Right) Invariant mass of the $p K \pi$ system. Again, points are for right $l \Lambda_{c}$ combinations and histogram for wrong sign combinations.

is measured.

\section{3. $B^{0}-\bar{B}^{0}$ Oscillations}

$B^{0}-\bar{B}^{0}$ transitions are allowed in the Standard Model via higher order weak interaction diagrams. Since the flavour eigenstates are not exactly the mass eigentstates, a $B^{0}$ produced at time $\tau=0$ has a certain probability to turn (mix) into a $\bar{B}^{0}$ at a later time $\tau$. Defining $x=\Delta m . \tau_{B}$, where $\Delta m$ is the mass difference and $\tau_{B}$ the average lifetime of the eigenstates of the mass matrix, the mixing probability is given by

$$
\mathcal{P}\left(B^{0}(0) \rightarrow \bar{B}^{0}(\tau)\right)=\frac{e^{-\tau / \tau_{B}}}{2 \tau_{B}} \cdot\left(1-\cos \left(x \frac{\tau}{\tau_{B}}\right)\right)
$$

The mixing parameters $x_{d, s}$, for the $B_{d, s}^{0}$ mesons, are related directly to the elements $V_{t(d, s)}$ of the CKM matrix.

In general, a time dependent mixing analysis requires knowledge of the flavour of the $B$ meson at production and decay times. Experimentally, to measure the decay time implies the use of some kind of vertexing algorithm. The flavour at the decay time is determined from the decay products. All the analyses reported here use semileptonic $B$ decays. The $B$ meson decay vertex is measured at the intersection of the lepton and reconstructed charm trajectories. The charm signal " $D$ " can be fully reconstructed as in Subsection 2.2, or inclusively tagged using a secondary vertex algorithm. In analogy with the semi-exclusive $B$ lifetime analysis, the proper decay length is calculated using

$$
c \tau=L_{x y} \frac{M_{B}}{p_{t}^{l^{\prime \prime} D^{\prime \prime}}} \cdot K
$$

where $K$ is an average kinematical Monte Carlo correction factor. In all cases, the flavour at decay time is determined using the charge of the lepton.

More challenging is to know the flavour at production time. Several approaches are possible. One possibility is to use the charge correlations of the $B$ meson and other particles produced 
in the same jet (same-side tagging). These correlations are expected to appear in the fragmentation process or $B^{* *}$ decays. The second possibility is to look at the other $B$ meson in the event (opposite-side tagging), that can (for instance) also decay semileptonically, or using a jet charge algorithm.

We present herein three recent measurements of the $B_{d}$ frequency from CDF.

\section{1. $B-\bar{B}$ mixing in $l D^{(*)}$ events}

In this analysis the charm is reconstructed explicitly using the decays of Subsection 2.2. Tracks with impact parameters significantly displaced from the primary vertex are selected in order to decrease combinatorial backgrounds. The signals are identified as peaks in the invariant mass distributions similar to the ones shown in Figure 2.

Same-side tagging is used. The momentum of the $B$ meson is approximated by the momentum of its reconstructed portion. Charged tracks within a cone around the reconstructed $B$ meson and consistent with the hypothesis that they originate from the primary vertex of the event are considered. Of the candidate tracks we select as the tag the track with minimum $p_{t}^{\text {rel }}$ relative to the sum of the momenta of the $B$ and that track. The efficiency for finding a tag is about $72 \%$.

Next, the number of right-sign (RS) correlations (i.e. $B^{0} \pi^{+}, B^{+} \pi^{-}$) is compared to the number of wrong sign correlations (WS) (i.e. $B^{0} \pi^{-}, B^{+} \pi^{+}$) as a function of the decay proper time. For the $B^{0}$ meson the following asymmetry is expected:

$$
A(\tau)=\frac{N_{R S}(\tau)-N_{W S}(\tau)}{N_{R S}(\tau)+N_{W S}(\tau)}=D \cos \left(\Delta m_{d} \tau\right)
$$

where $D$ is the so-called dilution of the tagging algorithm. $D$ is related to the mistag fraction $w$ by $D=1-2 w$. After correcting for channel cross-talk (as explained in Subsection 2.2), the values of $D$ and $\Delta m_{d}$ are extracted from a fit to the data. The results are shown in Figure 5 (left):

$$
\Delta m_{d}=0.45 \pm 0.06 \text { (stat.) } \pm 0.03 \text { (syst.) } \mathrm{ps}^{-1}
$$

and an effective tagging efficiency $\epsilon D^{2}=3.4 \pm 1.0 \pm 1.1 \%$. The systematic error is dominated by the uncertainty in the fraction of $D^{* *}$ in semileptonic $B$ decays.

\section{2. $B-\bar{B}$ mixing in $e-\mu$ events}

For this analysis, we trigger on leptons from the semileptonic decay of both $B$ hadrons in an event: $B_{1} \rightarrow e X$ and $B_{2} \rightarrow \mu X$. Sequential decays from one $B$ hadron are rejected with the requirement $M_{e \mu}>5 \mathrm{GeV}$. The charge of the lepton associated to the displaced vertex gives the flavour at the decay time. The other lepton provides the flavour tag at the production time.

An inclusive secondary vertex is reconstructed in association with one of the leptons. The vertexing algorithm has been tuned for high efficiency near $c \tau=0$, with the efficiency reaching a plateau of about $40 \%$ for $c \tau>500 \mu \mathrm{m}$. The boost resolution is about $22 \%$.

The challenge of this analysis is to determine the sample composition. It can estimated from several kinematical quantities, like $p_{t}^{r e l}$ or the invariant mass of the tracks that form the displaced vertex. Here $p_{t}^{r e l}$ is defined as the transverse momentum with respect to the lepton direction of the hardest track in a cone around the lepton. About $86 \%$ of the sample is made of $b \bar{b}$ events $(10-15 \%$ of these events contain sequential leptons), around $11 \%$ are events with at least a fake lepton, and the rest comes from $c \bar{c}$ events. 
CDF PRELIMINARY

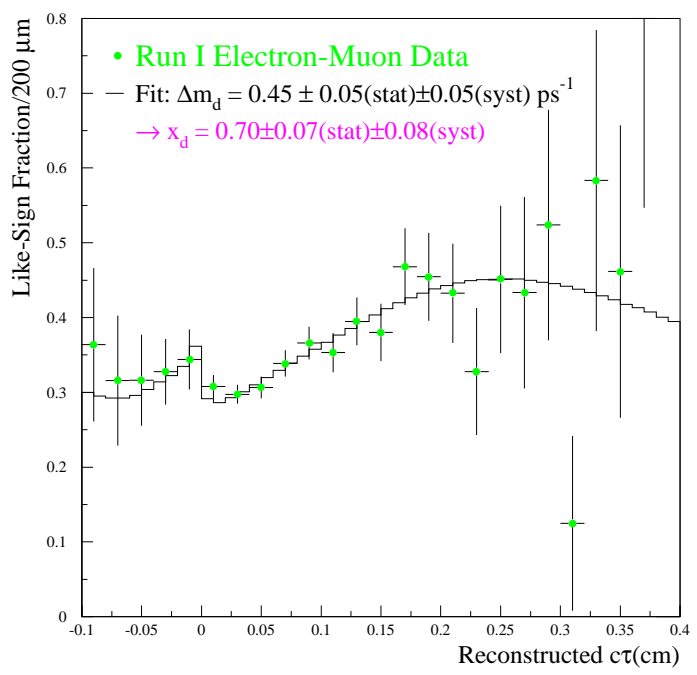

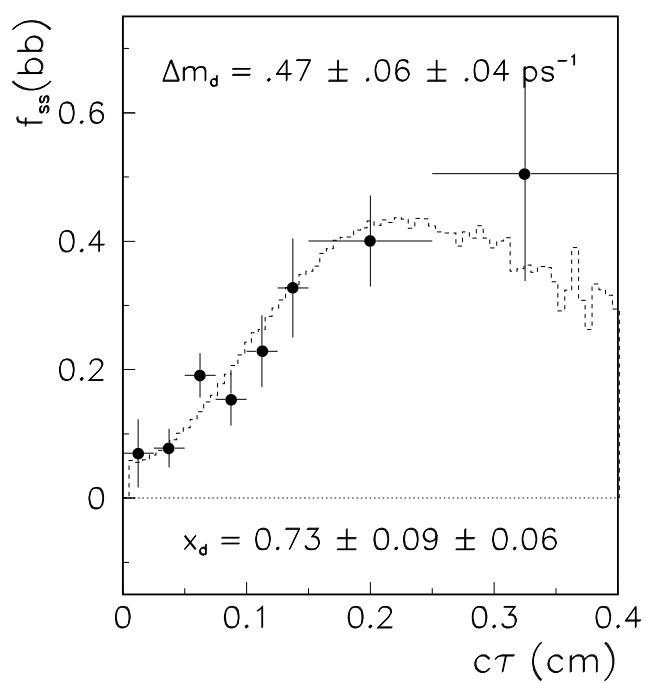

Fig. 4. Fitted like-sign fraction as a function of the decay proper time for the $e-\mu$ (left), and inclusive lepton (right) mixing analyses.

The final sample is formed by 6025 events with a secondary vertex around the electron (electron tags) and 5819 muon tags. Approximately $16 \%$ of these events, contain both an electron tag and a muon tag. Figure 4 (left) shows the dependence on $c \tau$ of the like-sign fraction of events, defined as $N_{L S}(\tau) /\left(N_{L S}(\tau)+N_{O S}(\tau)\right)$. A fit to the data is performed including components for direct and sequential $b$ decays, $c \bar{c}$, and fake events. The result is:

$$
\Delta m_{d}=0.45 \pm 0.05 \text { (stat.) } \pm 0.05 \text { (syst.) } \mathrm{ps}^{-1}
$$

where the dominant systematic error arises from the uncertainties in the sample composition.

\section{3. $B-\bar{B}$ mixing in inclusive lepton events}

There is still another CDF analysis that uses opposite-side tag. Starting from events that satisfy an inclusive lepton trigger with $p_{l}>8 \mathrm{GeV}$, opposite-side flavour tagging is implemented using jet charge and soft leptons. The result of this analysis (Figure 4 (right)) is:

$$
\Delta m_{d}=0.47 \pm 0.06 \text { (stat.) } \pm 0.04 \text { (syst.) } \mathrm{ps}^{-1}
$$

The effective tagging efficiency of this algorithm has been studied in detail by CDF. Values of $\epsilon D^{2}=0.6 \pm 0.1 \%$ for soft muon tagging, $0.3 \pm 0.1 \%$ for soft electron tagging, and $1.0 \pm 0.3 \%$ for the jet charge algorithm (after including impact parameter track information) are found respectively.

After taking into account the statistical overlap between the samples and common systematic errors, the CDF average result is

$$
\Delta m_{d}=0.454 \pm 0.033 \text { (stat.) } \pm 0.024 \text { (syst.) } \mathrm{ps}^{-1}
$$

This number is competitive to the LEP measurements ${ }^{9}$.

\section{Perspectives for Run 2}

In 1999, the Tevatron together with the Main Injector is supposed to deliver $2 \mathrm{fb}^{-1}$ in two years. By then, the CDF ${ }^{10}$ detector will be upgraded with a new silicon vertex detector, 

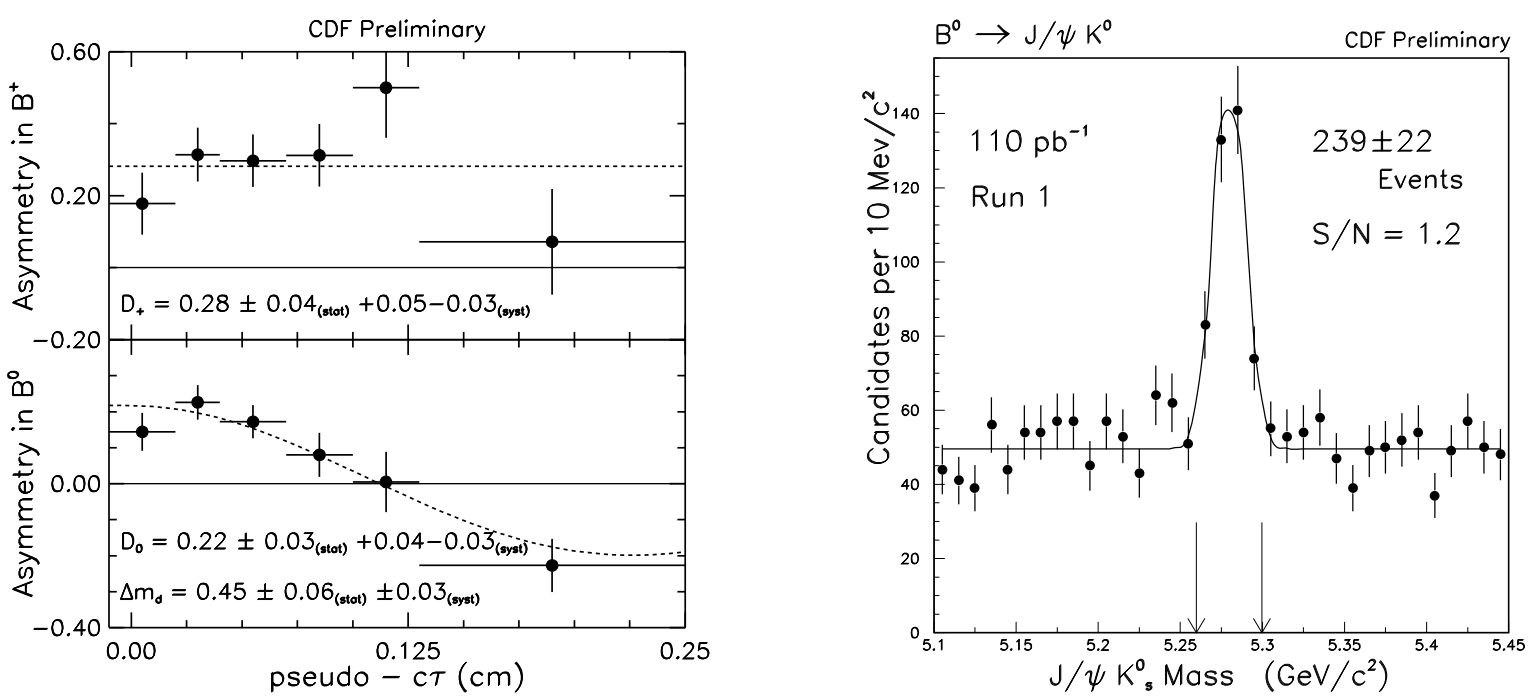

Fig. 5. (Left) Time dependent asymmetry for $B^{+}$(top) and $B^{0}$ mesons (bottom) in the $l D^{(*)}$ mixing analysis. (Right) $B^{0} \rightarrow J / \psi K_{S}^{0}$ signal collected at CDF during the 1992-96 run.

which doubles the fiducial volume of the current SVX and provides 3-d tracking. A new central tracking system with $\mathrm{dE} / \mathrm{dx}$ capabilities will be in place. With an upgraded trigger and DAQ system CDF plans to operate a fully hadronic trigger for the first time. D0 ${ }^{11}$ will enhance considerably its tracking capabilities with an inner silicon vertex detector, surrounded by four superlayers of a scintillating fiber tracker. These detectors will be located inside a 2 Tesla superconducting solenoid.

\section{1. $\sin 2 \beta$}

$\mathrm{CP}$ asymmetries in the decay $B^{0} \rightarrow J / \psi K_{s}^{0}$ determine the value of $\sin 2 \beta$. The $1992-96$ CDF signal (Figure 5 (right)) is formed by $239 \pm 22$ events with a signal-to-noise ratio of 1.2 . Taking into account the improvements in luminosity, lower trigger thresholds, etc, CDF expects to collect 15,000 such events. This results in an error of $\Delta \sin 2 \beta=0.09$, assuming $\epsilon D^{2}=6 \%$.

\section{2. $\sin 2 \alpha$}

CP asymmetries in the decay $B^{0} \rightarrow \pi^{+} \pi^{-}$are related to the value of $\sin 2 \alpha$. Here, the challenge is to be able to trigger on this decay. CDF plans to be able require two charged tracks at $\mathrm{L} 1$, and use impact parameter information at L2 $(20 \mathrm{~Hz})$. About 10,000 events are expected, that will produce an uncertainty of $\Delta \sin 2 \alpha=0.10$ for $\epsilon D^{2}=6 \%$.

\section{3. $B_{s}$ mixing}

Here the reach on $\Delta m_{s}$ is limited by the proper time resolution. Monte Carlo studies show that the experiment will be sensitive to values up to $10 \mathrm{ps}^{-1}$.

\section{Conclusions}

CDF has produced very competitive measurements of the $B$ hadron lifetimes. Mixing results are still dominated by statistics. In the frame of these analyses, the feasibility of a number of flavour tagging techniques have been demonstrated for the first time at a hadron collider. For 
Run 2, CDF and D0 will be significantly upgraded. The experiments will focus on the discovery of $\mathrm{CP}$ violation in the $B$ sector.

1. S. Abachi et al. (D0 Collaboration), Fermilab preprint FERMILAB-Conf-96-248-E, paper submitted to the 28th International Conference on High Energy Physics, Warsaw, Poland, July 1996.

2. I.I. Bigi, Nuovo Cim. A 109 (1996) 713.

3. M. Neubert, Int. J. Mod. Phys. A11 (1996) 4173.

4. F. Abe et al. (CDF Collaboration), Phys. Rev. Lett. 72 (1994) 3456.

5. F Abe et al. (CDF Collaboration), Phys. Rev. Lett. 76 (1996) 4462.

6. J.D. Richman, Proceedings of the 28th International Conference on High Energy Physics, Warsaw, Poland, July 1996.

7. F. Abe et al. (CDF Collaboration), Phys. Rev. Lett. 77 (1996) 1945.

8. F. Abe et al. (CDF Collaboration), Phys. Rev. Lett. 77 (1996) 1439.

9. L. Gibbons, Proceedings of the 28th International Conference on High Energy Physics, Warsaw. Poland, July 1996.

10. F. A be et al. (CDF Collaboration), Fermilab preprint FERMILAB-Pub-96-390-E, 1996.

11. S. Abachi et al. (D0 Collaboration) PAC-Report, 1996. 